\title{
Phenotypic diversity and molecular identification of the most prevalent anastomosis group of Rhizoctonia solani isolated from diseased faba bean plants
}

\author{
Mohamed Maha Helmy ${ }^{1}$, Gado Emad ${ }^{2}$, El-Deeb Samir ${ }^{1}$, Mostafa Helmy Mostafa ${ }^{1, *}$ \\ ${ }^{1}$ Plant Pathology Department, Faculty of Agriculture, Ain Shams University, Cairo, Egypt \\ ${ }^{2}$ Biology Department, Faculty of Science, Taif University, Taif, Saudi Arabia
}

Email address:

mostafa_mohamed@agr.asu.edu.eg (H. M. Mostafa)

\section{To cite this article:}

Mohamed Maha Helmy, Gado Emad, El-Deeb Samir, Mostafa Helmy Mostafa. Phenotypic Diversity and Molecular Identification of the Most Prevalent Anastomosis Group of Rhizoctonia solani Isolated from Diseased Faba Bean Plants. American Journal of Life Sciences. Vol. 3, No. 1, 2015, pp. 47-55. doi: 10.11648/j.ajls.20150301.19

\begin{abstract}
One hundred and thirty one isolates of Rhizoctonia spp. were isolated from faba bean plants showing root rot and stem canker collected from different fields in Delta region of Egypt. Forty six isolates were found to be polynucleate and the remaining isolates were binucleates. According to morphological features of isolates, they were classified into 12 groups. Polynucleate isolates were identified as Rhizoctonia solani (Kühn). The most aggressive isolate of $R$. solani was identified according to sequences of ITS1-5.8S rDNA-ITS4 and the sequence was compared with Thanatephorus cucumeris (teleomorphic phase) and other $R$. solani (NCBI GenBank). Sequence and comparison revealed that this isolate is $R$. solani AG4-HGI. Anastomosis test carried out between molecular identified isolate and 11 randomly chosen isolates resembles all groups of polynucleate $R$. solani. All tested isolates were completely fused between each other indicating that the prevalent AG of $R$. solani on faba bean is AG4-HG1.
\end{abstract}

Keywords: Anastmosis Group, Rhizoctonia Solani AG4-HGI, PCR, Grouping of isolates, Phylogenetic Analysis

\section{Introduction}

Rhizoctonia solani (Kühn) \{telomorhp: Thanatephorus cucumeris (Frank) Donk\} is one of the most important soil borne pathogens It infects many plants including faba bean (Vicia faba L.), causes very serious disease, ranging from damping-off to root rot and stem canker and may causes plant death (Salt, 1982; Lamari and Bernier, 1985; Omar, 1986; Elwakil et al., 2009). Owing to the phenotypic diversity of $R$. solani isolates, it is considered that this fungus comprises "group" and led to a classification system based on anastmosis grouping (AG). This AG is comprised of isolates among which perfect hyphal fusion occurs. This classification was supported by molecular sequencing of ITS5.8S rDNA-ITS4 (Kuninaga et al., 1997; Gonzales et al., 2001; Fenille et al., 2003).

This study was aimed to study morphological diversity among $R$. solani isolates and molecular identification of the most prevalent AG on faba bean in Egypt.

\section{Materials and Methods}

\subsection{Isolation and Identification of Rhizoctonia spp.}

Samples of diseased faba bean plants that showed root-rot and stem canker were obtained from different fields of Qualyobia and Giza Governorate. Procedure of isolation was carried out as mentioned earlier (Maha H. Mohamed et al., 2014). Identification of isolated Rhizoctonia spp. was carried out according to the procedure adopted by Sneh et al. (1991).

\subsection{Staining of Nuclei}

All isolates of Rhizoctonia spp. were grown individually on glass slide covered by water agar. Growing hyphae were stained by trypan blue to visualize the nuclei content especially in the apical compartment of fungal hyphae (Burpee et al., 1978) Stained hyphae were examined under light microscopy (400X) in order to count the number of nuclei/apical compartment then photographed. 


\subsection{Grouping of Isolates}

According to morphological features of Rhizoctonia spp. isolates i.e type of growth, density of aerial hyphae, mycelial color (melanization), density, distribution of sclerotia and number of nuclei/ apical compartments, all isolates were divided into groups. Every group contained approximately similar isolates.

\subsection{Molecular Identification of Rhizoctonia solani}

\subsubsection{DNA Extraction}

The most aggressive isolate on faba bean cotyledons (data not recorded) and identified as Rhizoctonia solani according to its morphological features (Singh et al., 1991) was grown in $100 \mathrm{ml}$ potato sucrose broth in $250 \mathrm{ml}$ flasks inoculated with $0.5 \mathrm{~cm}$ mycelial plugs. Flasks were incubated at $25 \pm 1^{\circ} \mathrm{C}$ for five days in the dark. Fungal harvesting was carried out by filtering through Whatman No.1 filter paper. The mycelium was ground in liquid nitrogen and total DNA was extracted according to the protocol of GeneJET Plant genomic DNA purification Kit (Thermo) \#K0791 (KuramaeIzioka, 1997).

\subsubsection{Polymerase Chain Reaction for Nucleotide Sequencing}

Amplification of the nuclear rDNA ITS region including the $5.8 \mathrm{~S}$ rDNA was performed with primers ITS4 and ITS5 (White et al., 1990). PCR reactions consisted of $25 \mu 1$ volume containing $2 \mu \mathrm{l}$ genomic DNA $(5-10 \mu \mathrm{g} / \mu \mathrm{l}), 2.5 \mu \mathrm{l}$ 10X PCR buffer (QIAGEN), $5 \mu$ l Q-solution (QIAGEN), 0.5 $\mu \mathrm{l}$ dNTPs (10 mM, Fermentas $\mathrm{GmbH}), 1.75 \mu \mathrm{l}$ of each primer $(10 \mu \mathrm{M}), 0.15 \mu \mathrm{l}$ Taq polymerase $(5$ units $/ \mu 1$, Fermentas $\mathrm{GmbH}$ ) and $11.35 \mu$ l sterile highly purified water. PCR was run in a Mastercycler Gradient (Eppendorf) with an initial denaturation step at $94^{\circ} \mathrm{C}$ for $10 \mathrm{~min}$ followed by 35 cycles at these conditions: $1 \mathrm{~min}$ at $94^{\circ} \mathrm{C}, 1 \mathrm{~min}$ at $55 \mathrm{C}$ and 1 min at $72^{\circ} \mathrm{C}$, with a final extension step at $72^{\circ} \mathrm{C}$ for $10 \mathrm{~min}$ by using Maxima Hot Start PCR Master Mix (Thermo)\#K0221.

\subsubsection{Sequencing and ITS Sequence Analysis}

Sequencing of PCR product was carried out on GATC Company by use ABI 3730 xI DNA sequencer by using forward and reverse primers. All these procedures were carried out in Sigma Scientific Service Technical Support, Sigma Scientific Services Co.

\subsubsection{Phylogenetic Analyses}

Sequences obtained in the present study and some available at GenBank were aligned with the multiple alignment program Clustal X (Thompson et al., 1997). A phylogenetic tree was constructed from distance matrix values by the neighbor joining method (Saitou and Nei, 1987).

\subsection{Anastomosis Test}

Anastomosis test was carried out between the well identified isolate of $R$. solani and eleven isolates randomly chosen from all groups of polynucleate isolates. Test was carried out by culturing each pair of the isolates on glass slide covered by water agar. Types of fusion between every pair was examined using light microscopy (400X).

\section{Results}

A total of 131 isolates from faba bean plants showed typical symptoms of root rot were isolated. Source of isolates and plant age from which the fungus was isolated are presented in Table (1).

Table 1. Origin of tester isolates of Rhizoctonia used in this study

\begin{tabular}{llll}
\hline $\begin{array}{l}\text { Source of } \\
\text { isolates }\end{array}$ & $\begin{array}{l}\text { Age of } \\
\text { Plants }\end{array}$ & $\begin{array}{l}\text { Number of samples with } \\
\text { root rot-like symptoms }\end{array}$ & $\begin{array}{l}\text { Number of } \\
\text { isolates }\end{array}$ \\
\hline El-Kaliobia & seedling & 10 & 18 \\
El-Kaliobia & adult & 77 & 80 \\
El-Sharkia & adult & 40 & 17 \\
El-Giza & adult & 20 & 16 \\
Total & & 147 & 131 \\
\hline
\end{tabular}

\subsection{Identification of Rhizoctonia}

\subsubsection{Morphological and Cultural Characteristics}

Morphological studies of isolated Rhizoctonia spp. revealed that 46 isolates fitted well with $R$. solani characteristics according to criteria adopted by Sneh et al. (1991), which include number of nuclei, production of sclerotium and brown pigmentation, branching near distal septum, constriction of the branch base of hyphae and formation of septa within a short distance of the origin of branches. For determination of number of nuclei/apical compartment, colonies of Rhizoctonia spp. were stained with trypan blue. Forty six isolates were polynucleate containing 3 to 8 nuclei/apical compartment or moniloid hyphal cell (Fig. 1). Cultural characteristics such as colony diameter, morphology of mycelial growth and degree of pigmentation showed great diversity among the total of 131 isolates (Table 2) and Fig. (1). Isolates of Rhizoctonia are classified to twelve groups according to colonies' morphological characters. Seven of them belong to polynucleate Rhizoctonia and 5 groups belong to binucleate Rhizoctonia. Average of growth rate \pm standard deviation was calculated. As presented in Table (2), polynucleate isolates have in general the higher rate of growth. Degree of pigmentation also varied between groups. Polynucleate Rhizoctonia are more pigmented than binucleate one.
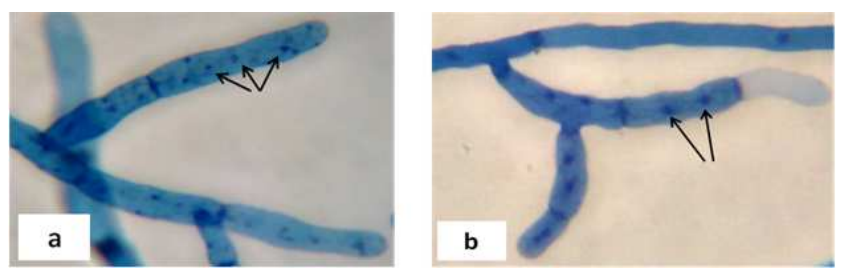

Fig. 1. Hyphae of Rhizoctonia solani showing polynucleate (a) or/ binucleate (b) stained with trypan blue.Arrows indicate nuclei. 
Table 2. Radial colony growth, degree of melanin pigmentation production and number of nuclei of Rhizoctonia isolates growing on PSA medium; degree of pigmentation was determined according to Kim et al. (2001)

\begin{tabular}{|c|c|c|c|c|}
\hline Isolates & $\begin{array}{l}\text { Colony } \\
\text { diameter (cm) }\end{array}$ & $\begin{array}{l}\text { degree of } \\
\text { melanin } \\
\text { pigmentation }\end{array}$ & $\begin{array}{l}\text { Source } \\
\text { of isolate }\end{array}$ & $\begin{array}{l}\text { Number } \\
\text { of nuclei }\end{array}$ \\
\hline \multicolumn{5}{|l|}{ Group 1} \\
\hline Gf5 & 8.76 & 3 & G & M \\
\hline Gf6 & 8.65 & 3 & $\mathrm{G}$ & M \\
\hline Gf7 & 8.41 & 3 & $\mathrm{G}$ & $\mathrm{M}$ \\
\hline Gf8 & 8.50 & 3 & G & M \\
\hline Gf9 & 8.85 & 3 & $\mathrm{G}$ & M \\
\hline A2 & 7.16 & 3 & $\mathrm{~K}$ & M \\
\hline A3 & 8.86 & 3 & $\mathrm{~K}$ & M \\
\hline A4 & 7.75 & 3 & K & M \\
\hline A7 & 6.36 & 3 & K & M \\
\hline $\mathrm{H} 10$ & 7.25 & 3 & S & M \\
\hline SD & $8.05 \pm 0.87$ & & & \\
\hline \multicolumn{5}{|l|}{ Group 2} \\
\hline S1 & 7.05 & 2 & $\mathrm{~K}$ & M \\
\hline Gf1 & 8.81 & 2 & G & M \\
\hline Gf2 & 9.00 & 2 & G & M \\
\hline H11 & 9.00 & 2 & S & M \\
\hline $\mathrm{H} 12$ & 9.00 & 2 & S & M \\
\hline H13 & 9.00 & 2 & $\mathrm{~S}$ & M \\
\hline H14 & 8.80 & 2 & S & M \\
\hline H15 & 7.73 & 2 & S & M \\
\hline M9 & 9.00 & 2 & K & M \\
\hline SD & $8.59 \pm 0.71$ & & & \\
\hline \multicolumn{5}{|l|}{ Group 3} \\
\hline GB3 & 4.23 & 2 & G & M \\
\hline GB4 & 5.25 & 2 & G & M \\
\hline GB5 & 5.03 & 2 & G & M \\
\hline GB6 & 5.68 & 2 & G & M \\
\hline GB7 & 4.78 & 2 & G & M \\
\hline GB8 & 4.10 & 2 & G & M \\
\hline H16 & 8.03 & 2 & S & M \\
\hline H17 & 8.78 & 2 & S & M \\
\hline SD & $1.73 \pm 5.73$ & & & \\
\hline \multicolumn{5}{|l|}{ Group 4} \\
\hline A1 & 8.06 & 2 & $\mathrm{~K}$ & M \\
\hline A5 & 8.30 & 2 & K & M \\
\hline $\mathrm{S} 2$ & 8.23 & 2 & $\mathrm{~K}$ & M \\
\hline S3 & 5.33 & 2 & K & M \\
\hline $\mathrm{S} 4$ & 4.30 & 2 & K & M \\
\hline GB1 & 3.76 & 2 & G & M \\
\hline B8 & 4.18 & 2 & $\mathrm{~K}$ & M \\
\hline B10 & 3.23 & 2 & K & M \\
\hline B11 & 4.35 & 2 & $\mathrm{~K}$ & M \\
\hline B12 & 3.83 & 2 & $\mathrm{~K}$ & M \\
\hline SD & $2.07 \pm 5.52$ & & & \\
\hline \multicolumn{5}{|l|}{ Group 5} \\
\hline Mo1 & 4.60 & 2 & K & M \\
\hline Mo2 & 5.46 & 2 & K & M \\
\hline Mo3 & 6.01 & 2 & K & M \\
\hline Mo4 & 5.43 & 2 & $\mathrm{~K}$ & M \\
\hline Mo5 & 5.75 & 2 & K & M \\
\hline Gf3 & 5.96 & 2 & G & M \\
\hline Gf4 & 6.51 & 2 & G & M \\
\hline SD & $0.59 \pm 5.67$ & & & \\
\hline
\end{tabular}

\begin{tabular}{|c|c|c|c|c|}
\hline & & Group 6 & & \\
\hline \multirow[t]{2}{*}{$\mathrm{Cu}$} & 7.20 & 2 & K & M \\
\hline & & Group 7 & & \\
\hline \multirow[t]{2}{*}{ H7 } & 5.75 & 0 & S & M \\
\hline & & Group 8 & & \\
\hline A15 & 2.51 & 0 & K & B \\
\hline A16 & 3.00 & 0 & $\mathrm{~K}$ & B \\
\hline A17 & 3.25 & 0 & K & B \\
\hline A18 & 3.68 & 0 & K & B \\
\hline A20 & 3.06 & 0 & K & B \\
\hline A21 & 4.45 & 0 & K & B \\
\hline A 22 & 3.11 & 0 & K & B \\
\hline A23 & 3.35 & 0 & K & B \\
\hline M1 & 3.21 & 0 & K & B \\
\hline M2 & 3.98 & 0 & K & B \\
\hline M3 & 4.86 & 0 & K & B \\
\hline M4 & 3.05 & 0 & K & B \\
\hline M5 & 3.31 & 0 & K & B \\
\hline M6 & 4.25 & 0 & K & B \\
\hline M7 & 3.43 & 0 & K & B \\
\hline M8 & 5.42 & 0 & K & B \\
\hline M11 & 3.75 & 0 & K & B \\
\hline M12 & 4.23 & 0 & K & B \\
\hline M13 & 3.13 & 0 & K & B \\
\hline H1 & 5.10 & 0 & S & B \\
\hline $\mathrm{H} 2$ & 5.98 & 0 & S & B \\
\hline H3 & 5.18 & 0 & S & B \\
\hline $\mathrm{H} 4$ & 5.90 & 0 & S & B \\
\hline H5 & 4.55 & 0 & S & B \\
\hline H6 & 5.16 & 0 & S & B \\
\hline H8 & 5.85 & 0 & $\mathrm{~S}$ & B \\
\hline H9 & 5.33 & 0 & S & B \\
\hline S5 & 5.10 & 0 & K & B \\
\hline S6 & 5.35 & 0 & K & B \\
\hline S7 & 4.96 & 0 & K & B \\
\hline S8 & 2.90 & 0 & K & B \\
\hline S9 & 4.31 & 0 & K & B \\
\hline $\mathrm{S} 10$ & 3.40 & 0 & K & B \\
\hline S11 & 4.10 & 0 & K & B \\
\hline $\mathrm{S} 12$ & 5.26 & 0 & K & B \\
\hline $\mathrm{S} 13$ & 2.96 & 0 & K & B \\
\hline S14 & 4.91 & 0 & K & B \\
\hline S15 & 5.36 & 0 & K & B \\
\hline S16 & 3.73 & 0 & K & B \\
\hline S18 & 2.95 & 0 & K & B \\
\hline S19 & 4.00 & 0 & K & B \\
\hline B5 & 6.25 & 0 & K & B \\
\hline B6 & 5.76 & 0 & K & B \\
\hline B7 & 5.21 & 0 & K & B \\
\hline Mo6 & 6.46 & 0 & K & B \\
\hline Mo7 & 6.45 & 0 & K & B \\
\hline SD & $1.11 \pm 4.38$ & & & \\
\hline \multicolumn{5}{|c|}{ Group 9} \\
\hline A6 & 6.86 & 2 & K & B \\
\hline A9 & 3.03 & 2 & K & B \\
\hline A10 & 9.00 & 2 & K & B \\
\hline A11 & 5.31 & 2 & K & B \\
\hline A12 & 6.56 & 2 & K & B \\
\hline A13 & 3.50 & 2 & K & B \\
\hline A14 & 3.26 & 2 & K & B \\
\hline
\end{tabular}




\begin{tabular}{|c|c|c|c|c|}
\hline SD & \multicolumn{4}{|c|}{$2.24 \pm 5.36$} \\
\hline \multicolumn{5}{|c|}{ Group 10} \\
\hline M14 & 7.20 & 2 & $\mathrm{~K}$ & B \\
\hline M15 & 8.05 & 2 & $\mathrm{~K}$ & B \\
\hline M16 & 6.95 & 2 & $\mathrm{~K}$ & B \\
\hline M17 & 7.61 & 2 & $\mathrm{~K}$ & B \\
\hline M18 & 7.30 & 2 & $\mathrm{~K}$ & B \\
\hline M19 & 6.81 & 2 & $\mathrm{~K}$ & B \\
\hline M20 & 6.11 & 2 & K & B \\
\hline M21 & 4.38 & 2 & $\mathrm{~K}$ & B \\
\hline M22 & 6.96 & 2 & $\mathrm{~K}$ & B \\
\hline M23 & 5.86 & 2 & $\mathrm{~K}$ & B \\
\hline M24 & 6.88 & 2 & $\mathrm{~K}$ & B \\
\hline M25 & 7.08 & 2 & $\mathrm{~K}$ & B \\
\hline M26 & 7.65 & 2 & $\mathrm{~K}$ & B \\
\hline M27 & 8.15 & 2 & $\mathrm{~K}$ & B \\
\hline M28 & 6.95 & 2 & $\mathrm{~K}$ & B \\
\hline M29 & 5.66 & 2 & $\mathrm{~K}$ & B \\
\hline M30 & 6.51 & 2 & $\mathrm{~K}$ & B \\
\hline M31 & 7.05 & 2 & $\mathrm{~K}$ & B \\
\hline M32 & 7.10 & 2 & $\mathrm{~K}$ & B \\
\hline M33 & 6.50 & 2 & $\mathrm{~K}$ & B \\
\hline M34 & 6.93 & 2 & $\mathrm{~K}$ & B \\
\hline M35 & 8.23 & 2 & $\mathrm{~K}$ & B \\
\hline M36 & 6.50 & 2 & $\mathrm{~K}$ & B \\
\hline M37 & 6.55 & 2 & $\mathrm{~K}$ & B \\
\hline B2 & 6.01 & 2 & $\mathrm{~K}$ & B \\
\hline A8 & 7.30 & 2 & $\mathrm{~K}$ & B \\
\hline \multirow[t]{2}{*}{ SD } & 6.85 & & & \\
\hline & & & & \\
\hline M10 & 9.00 & 1 & $\mathrm{~K}$ & B \\
\hline B1 & 7.56 & 1 & $\mathrm{~K}$ & B \\
\hline \multirow[t]{2}{*}{ SD } & 8.28 & & & \\
\hline & & & & \\
\hline D & 6.66 & 2 & $\mathrm{~K}$ & B \\
\hline
\end{tabular}

- Colony diameter $(\mathrm{cm})$ between isolates average \pm standard deviation $(\mathrm{SD})$. - Radial colony growth was recorded after 48 hours.

-melanin pigmentation production was a degree recorded after two weeks. - G: El-Giza, S: El-Sharkia, K: El-Kaliobia.

- B: binuclear, M: multinuclear.

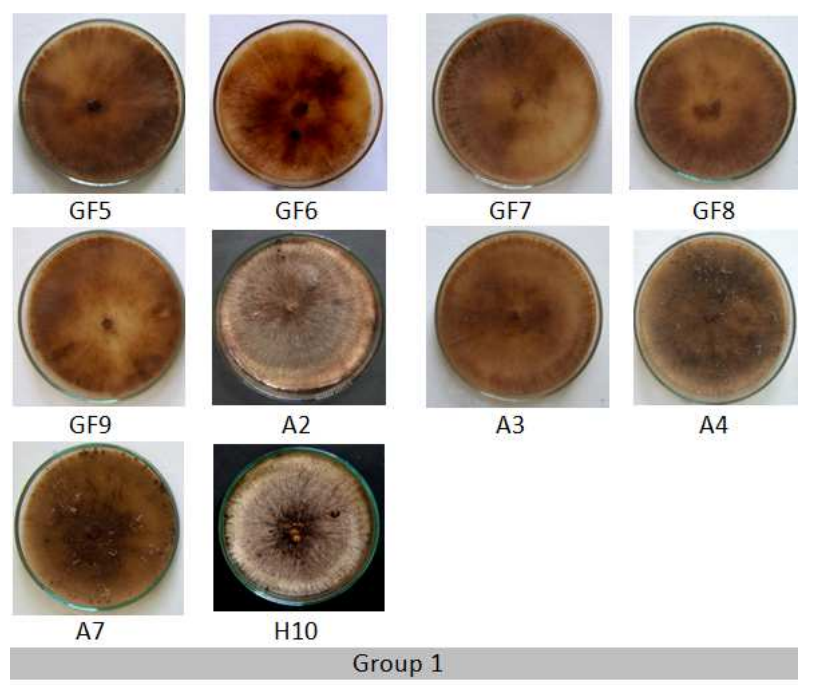

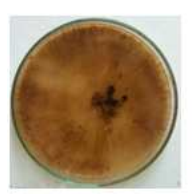

S1

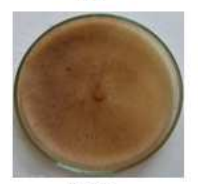

$\mathrm{H} 12$

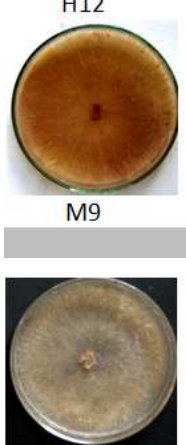

GB3
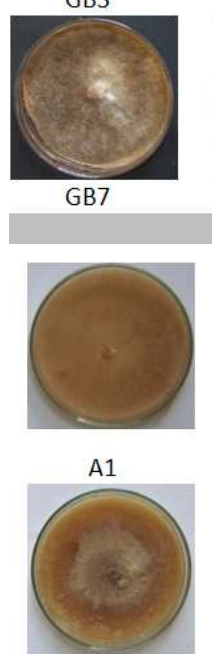

S4

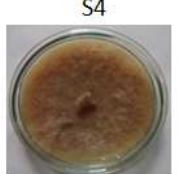

B11

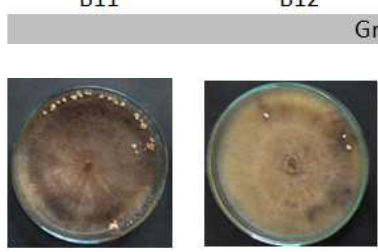

M01

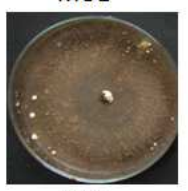

M05

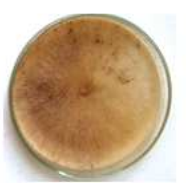

GF1

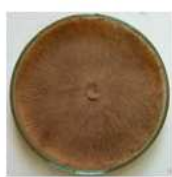

H13

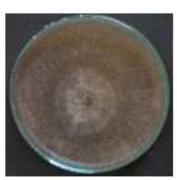

GB4

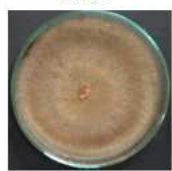

GB8

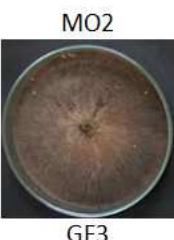

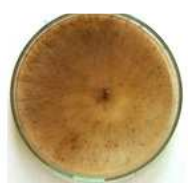

GF2

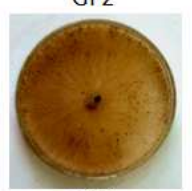

$\mathrm{H} 14$

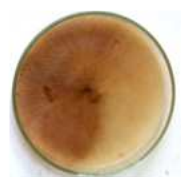

H11

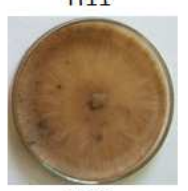

H15 Group 2

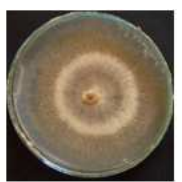

GB5

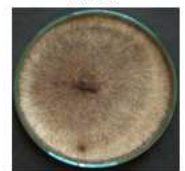

H16

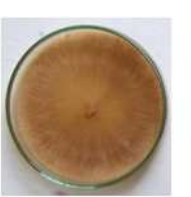

S2

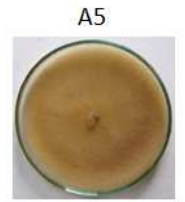

GB1
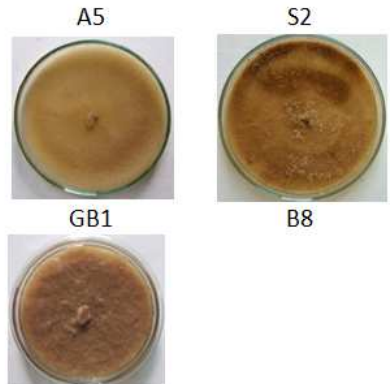

B8

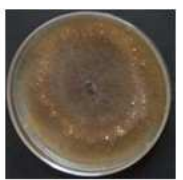

GB6

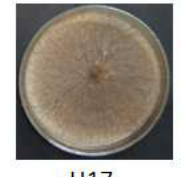

H17
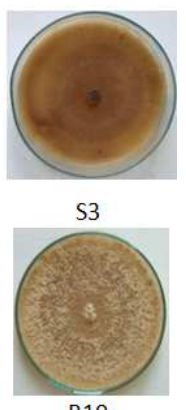

B10
$\mathrm{MO} 3$
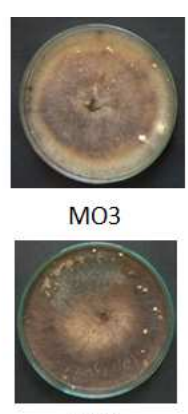

GF4

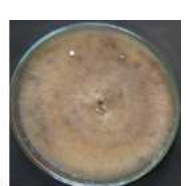

$\mathrm{MO} 4$ 

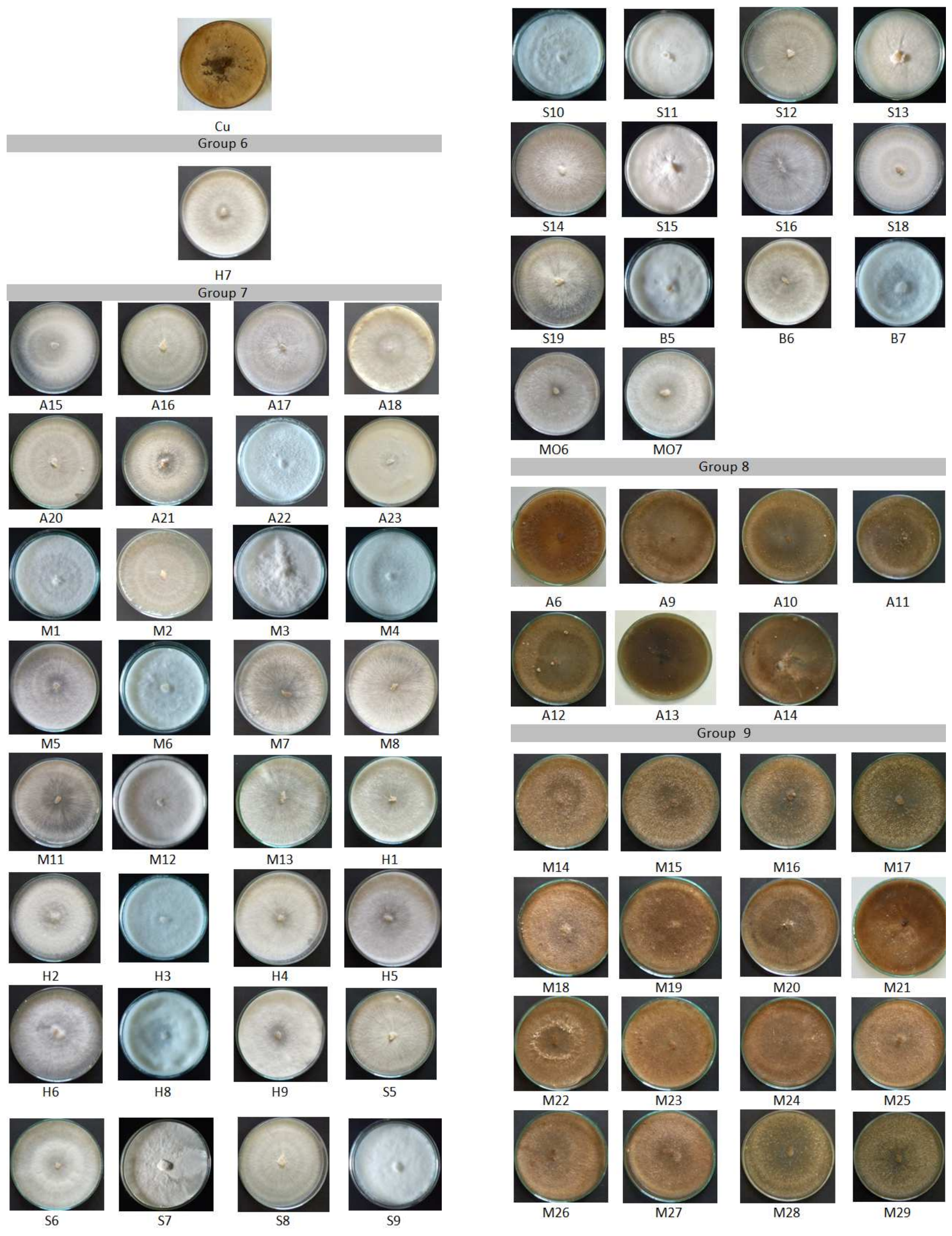

M16
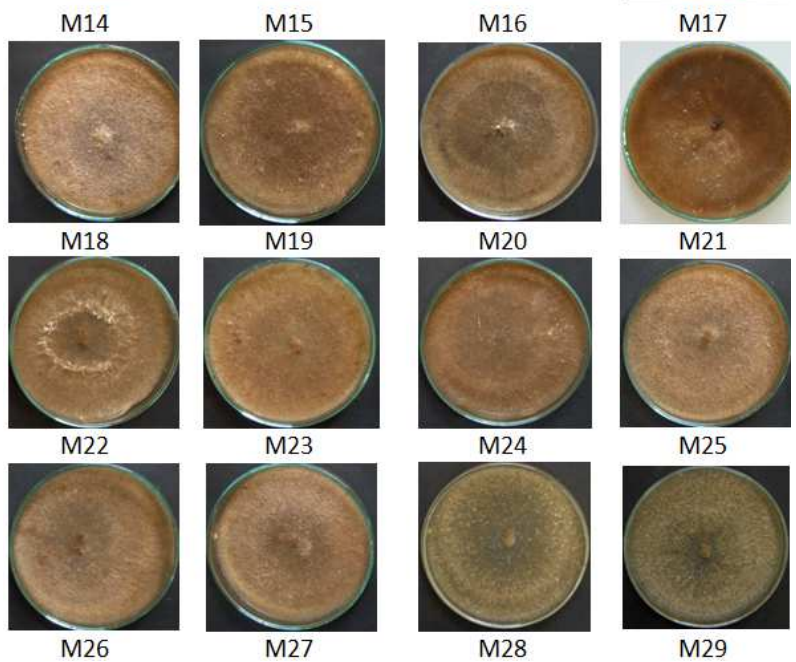

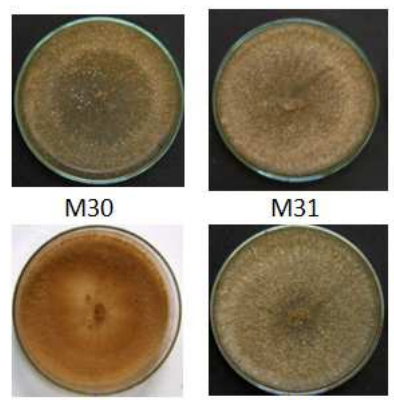

M34

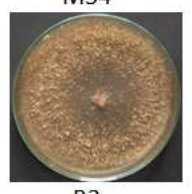

B2

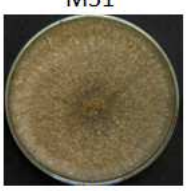

M35

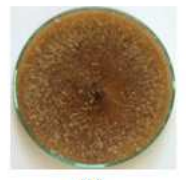

A8

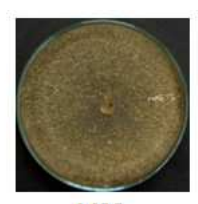

M32
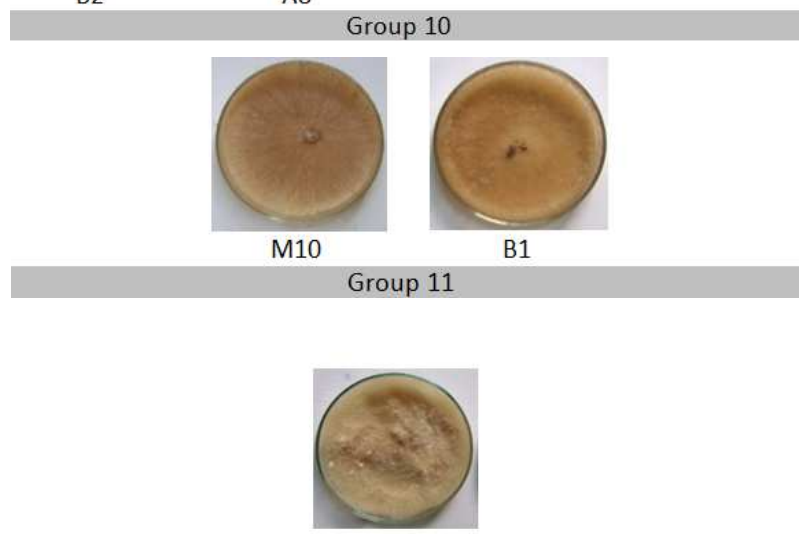

D

Group 12

Fig. 2. Grouping of R. solani isolates depend on cultural characteristics.

\subsubsection{Molecular Identification}

DNA of the most aggressive faba bean isolate was amplified using universal primers ITS1 and ITS4 using PCR technique. Primers generated a fragment of approximately $700 \mathrm{bp}$ long. The whole ITS region (ITS1, 5.8S rDNA and
ITS4 of this isolate was sequenced in both directions with the same primers used for PCR amplifications. Sequences were aligned (Fig. 3).

Comparison of whole region (ITS1, 5.8S rDNA and ITS4) of isolate under study with that of Thanatophorus cucumeris (teleomorph) and other $R$. solani (anamorph) (NCBI GenBank) revealed that the similarity in nucleotide sequence between them reached 97\% similarity (Table.3) and (Fig. 4). According to these comparisons, the isolate under study is identified as $R$. solani AG4 and the whole region (ITS1, 5.8S rDNA and ITS4) was registered in NCBI GeneBank with accession number KF907731.1.

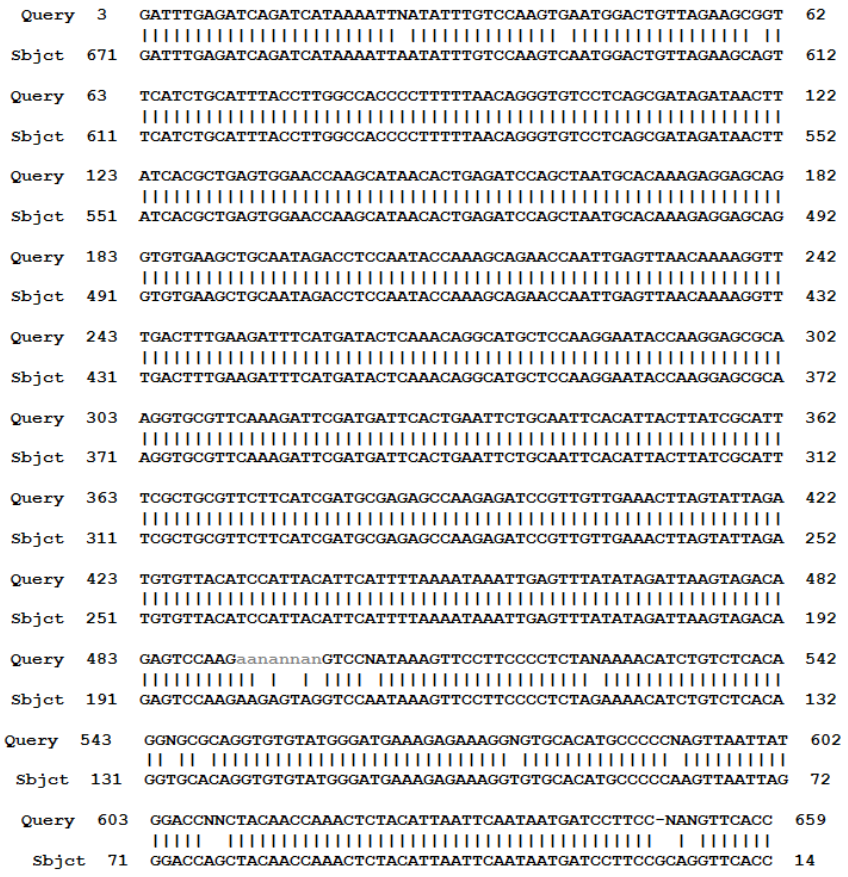

Fig. 3. Sequences aligned with other known R. solani sequences from the NCBI GenBank.

Table 3. Comparison of whole region (ITS1, 5.8S rDNA and ITS4) of isolate under study with that of Thanatophorus cucumeris (teleomorph) and other R. solani (anamorph) (NCBI GenBank) revealed the similarity in nucleotide sequence between them.

\begin{tabular}{|c|c|c|c|c|c|c|}
\hline Description & Max score & Total score & Query cover & E value & Ident & Accession \\
\hline Thanatophorus cucumeris isolate PT73 18S & 1119 & 1119 & $93 \%$ & 0.0 & $97 \%$ & JQ219361. 1 \\
\hline Rhizoctonia solani strain AG 4-HGI isolate LDDT01-1 18S & 1110 & 1110 & $93 \%$ & 0.0 & $97 \%$ & KF907731. 1 \\
\hline Rhizoctonia solani RT 14-2 & 1110 & 1110 & $93 \%$ & 0.0 & $97 \%$ & FJ746939. 1 \\
\hline Rhizoctonia solani RT 26-4 & 1106 & 1106 & $93 \%$ & 0.0 & $97 \%$ & FJ747974. 1 \\
\hline Thanatophorus cucumeris isolate $\mathrm{Rh}$ Faq & 1106 & 1106 & $92 \%$ & 0.0 & $98 \%$ & DQ021450. 1 \\
\hline Rhizoctonia solani isolate RS79 & 1101 & 1101 & $92 \%$ & 0.0 & $97 \%$ & JQ616856. 1 \\
\hline Rhizoctonia solani isolate RT 26-1 & 1101 & 1101 & $93 \%$ & 0.0 & $97 \%$ & FJ746973. 1 \\
\hline Thanitophorus cucumeris serain Rol 222 & 1101 & 1101 & $93 \%$ & 0.0 & $97 \%$ & AY684924. 1 \\
\hline Rhizoctonia solani AG4 HGI isolate IBRS01 & 1097 & 1097 & $93 \%$ & 0.0 & $97 \%$ & KF746162. 1 \\
\hline Thanitophorus cucumeris isolate CR-26 & 1097 & 1097 & $93 \%$ & 0.0 & $97 \%$ & JF699277. 1 \\
\hline Thanitophorus cucumeris isolate KT63-1 & 1097 & 1097 & $93 \%$ & 0.0 & $97 \%$ & EF203246. 1 \\
\hline Rhizoctonia solani strain 11D & 1095 & 1095 & $93 \%$ & 0.0 & $97 \%$ & JX294316. 1 \\
\hline Thanitophorus cucumeris & 1094 & 1094 & $93 \%$ & 0.0 & $97 \%$ & KJ715964. 1 \\
\hline Rhizoctonia solani isolate Remol & 1094 & 1094 & $92 \%$ & 0.0 & $98 \%$ & KC70958. 1 \\
\hline
\end{tabular}




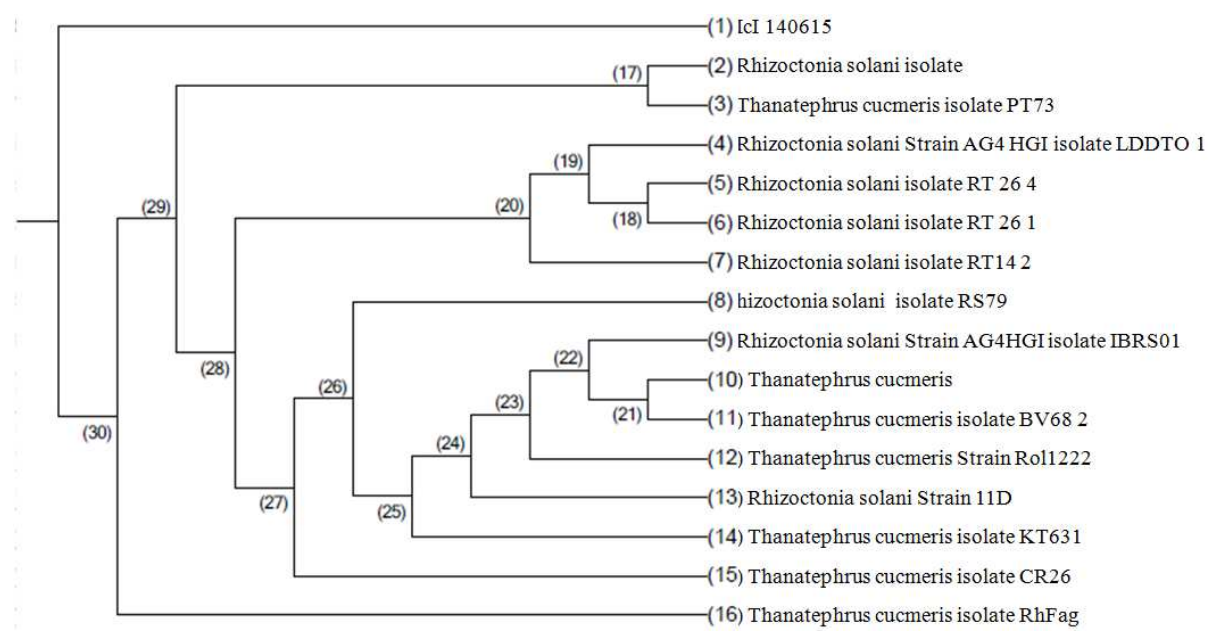

Fig. 4. Comparison of whole region (ITS1, 5.8S rDNA and ITS4) of isolate under study with that of Thanatophorus cucumeris (teleomorph) and other R. solani (anamorph) (NCBI GenBank)

Table 4. Hyphal fusion among chosen polynucleate Rhizoctonia isolates.

\begin{tabular}{|c|c|c|c|c|c|c|c|c|c|c|c|c|}
\hline Isolates & A3 & H10 & GF6 & S1 & GF1 & GF2 & H14 & M9 & GB5 & A5 & MO4 & $\mathbf{C u}$ \\
\hline $\mathrm{A} 3$ & + & + & + & + & + & + & + & + & + & + & + & + \\
\hline $\mathrm{H} 10$ & + & + & + & + & + & + & + & + & + & + & + & \\
\hline Gf6 & + & + & + & + & + & + & + & + & + & + & & \\
\hline $\mathrm{S} 1$ & + & + & + & + & + & + & + & + & + & & & \\
\hline GF1 & + & + & + & + & + & + & + & + & & & & \\
\hline GF2 & + & + & + & + & + & + & + & & & & & \\
\hline H14 & + & + & + & + & + & + & & & & & & \\
\hline M9 & + & + & + & + & + & & & & & & & \\
\hline GB5 & + & + & + & + & & & & & & & & \\
\hline A5 & + & + & + & & & & & & & & & \\
\hline MO4 & + & + & & & & & & & & & & \\
\hline $\mathrm{Cu}$ & + & & & & & & & & & & & \\
\hline
\end{tabular}

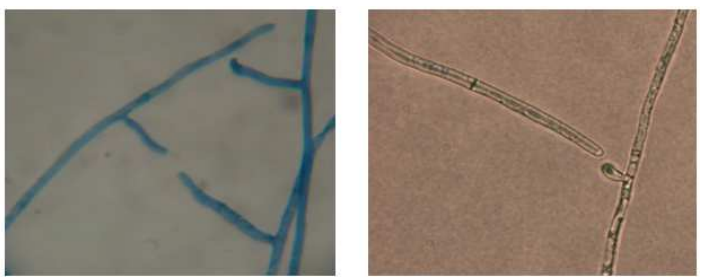

a: Hyphae of two isolates of Rhizoctonia solani attracted to each other

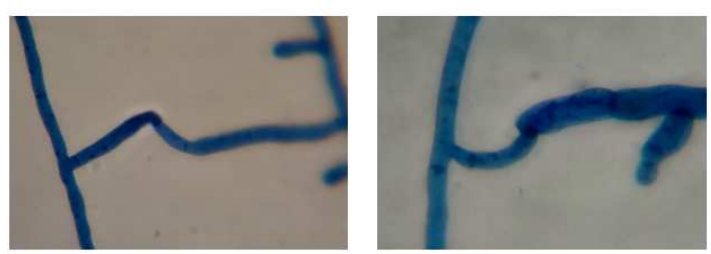

b: Perfect fusion between hyphal tips of two isolates of Rhizoctonia solani.

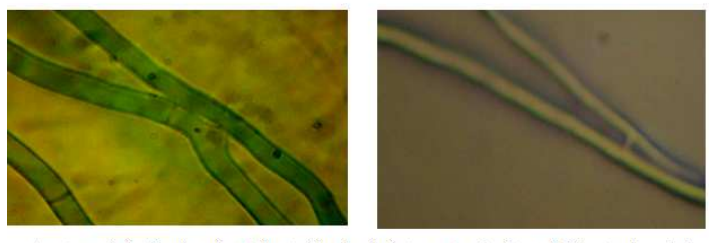

c: Anastomosis by forming short tube at side of cells between two isolates of Rhizoctonia solani.

Fig. 5. Examples of fusion between two different isolates.

\subsection{Anastomosis Test}

The accurate identified $R$. solani isolate was tested for fusion with another 11 isolates of polynucleate $R$. solani randomly chosen among polynucleate $R$. solani groups. As shown in Table (4). Data obtained indicated that all chosen tested isolates fused perfectly between them. Example of fusion are illustrated in Fig. $5 \mathrm{a}, \mathrm{b}$, and c). This result indicates clearly that the randomly chosen $R$. solani isolates belong to the same Anastomosis group (AG4 HGI).

\section{Discussion}

The fungus Rhizoctonia solani (Kühn) is reported to cause economic losses in many economical plants including faba bean causing damping off, root and hypocotyl rots and stem canker. The tremendous diversity in morphology and pathogenecity of $R$. solani has led to classification system based on anastomosis grouping (AG). This AG is comprised of isolates among which hyphal fusion occurs and this classification system has been supported by molecular evidence (Kuninaga and Yokosawa, 1982 a\&b, 1984, 1985; Vilgalys, 1988; Carling and Kuninaga, 1990; Kuninaga et al., 
1997; Gonzalez et al., 2001; Fenille et al., 2003).

In this study a total of 131 isolates of Rhizoctonia spp. were isolated from diseased faba bean plants collected from different provinces in Egypt. It is well established that genus Rhizoctonia classified according to the number of nuclei in the apical compartment into two groups: binucleate (in which apical compartments contains 2 nuclei) and polynucleate (in which apical compartments contain 3 to 11 nuclei). Data obtained indicated that binucleate Rhizoctonia comprise $64.88 \%$ from the total isolate, indicating that this group is dominates over polynucleate Rhizoctonia on faba bean plants. The behavior of binucleate Rhizoctonia and its effect on plants and its host range has not received adequate attention.

Isolated Rhizoctonia spp. was grouped into 12 groups according to their morphological characters, degree of melanization and number of nuclei. Polynucleate Rhizoctonia occupied 7 groups and binucleate ones occupied 5 groups. The diversities between polynucleate Rhizoctonia were very strong in comparison to binucleate groups and the divergence among polynucleate Rhizoctonia may be due to heterokaryosis and parasexual cycle within apical compartments resulting in these deviations in polynucleate Rhizoctonia spp. (Parmeter, et al., 1963).

Twelve isolates resembling polynucleate Rhizoctonia groups were identified according to their morphological features according to Sneh et al. (1991). According to these features, isolates were identified as Rhizoctonia solani (Kühn). Anastomosis test was carried out between all isolates by culturing pairs of isolates facing each other on glass slide covered by water agar in sterilized Petri dish. It was observed that all isolates were perfectly fused between each other. Microscopic observation proved that hyphal apicals were attracted to each other and then fused (tip -by- tip). Another fusion was observed (apical -to- hyphal side; side -by- side), indicating that all tested isolates belong to the same AG.

One of these tested isolates which had high pathogenic effect on faba bean was chosen for further identification by rDNA - ITS sequences. Data obtained indicated that the similarities between tested isolate and Thanatephorus cucumeris (teleomorph of $R$. solani) reached 97\% similarity and between tested isolate and $R$. solani isolates (anamorph) reached $97 \%$ similarities, indicating that the tested isolate is R. solani (Kühn) AG4-HG1.

\section{References}

[1] Burpee, L. L.; Sanders, P. L.; Cole, H. Jr. and Kim, S. H. (1978). A staining technique for nuclei of Rhizoctonia solani and related fungi. Mycologia 70: 1281-1283.

[2] Carling, D.E. and Kuninaga, S. (1990). DNA based-sequence homology in Rhizoctonia solani Kühn: inter-and intra-group relatedness of anastomosis group-9. Phytopathology 80:13621364.

[3] Elwakil, M. A.; El-Refai, I. M.; Awadallah, O. A.; ElMetwally, M. A. and Mohamed, M. S. (2009). Seed-borne pathogens of faba bean in Egypt: Detection and Pathogenicity. Plant Pathology Journal, 8(3): 90-97.
[4] Fenille, R. C.; Ciampi, M. B.; Kuramae, E. E. and Souza, N. L. (2003). Identification of Rhizoctonia solani Associated with Soybean in Brazil by rDNA-ITS Sequences. Fitopatologia Brasileira 28:413-419. 2003.

[5] Gonzalez, D.; Carling, D. E.; Kuninaga, S.; Vilgalys, R. And Cubeta, M. A. (2001). Rhibosomal DNA systematics of Ceratobasidium and Thanatephorus with Rhizoctonia anamorphs. Mycologia., 93: 1138-1150.

[6] Kim, H. T.; Chung, Y. R. and Cho, K. Y. (2001). Mycelial melanization of Rhizoctonia solani AG1 affected pathogenicity in rice. Plant Patho. J., 17 (4): 210-215.

[7] Kuninaga, S. and Yokosawa, R. (1982a). DNA based sequence homology in Rhizoctonia solani Kühn. I. Genetic relatedness within anastomosis group 1. Annals of the Phytopathological Society of Japan 48:659-667.

[8] Kuninaga, S. and Yokosawa, R. (1982b). DNA based sequence homology in Rhizoctonia solani Kühn. II. Genetic relatedness within anastomosis group 2. Annals of the Phytopathological Society of Japan 48:668-673.

[9] Kuninaga, S. and Yokosawa, R. (1984). DNA base-sequence homology in Rhizoctonia solani Kühn. IV. Genetic relatedness within AG-4. Ann Phytopathol Soc 50:322-330.

[10] Kuninaga, S. and Yokosawa, R. (1985). DNA base sequence homology in Rhizoctonia solani Kühn. VI. Genetic relatedness among seven anastomosis groups. Ann. Phytopath. Soc. Japan. 51: $127-132$.

[11] Kuninaga, S; Natsuaki, T.; Takeuchi, T. and Yokosawa, R. (1997). Sequence variation of the rDNA ITS regions within and between anastomosis groups in Rhizoctonia solani. Current Genetics., 32: 237-243.

[12] Kuramae-Izioka, E.E. (1997). A rapid. easy and high yield protocol for total genomic DNA isolation from Colletotrichum gloeosporioides and Fusarium oxysporum for RAPD. Revista Unimar 19:683-689.

[13] Lamari, L. and Bernier, C. C. (1985). Etiology of seedling blight and root rot of faba bean (Vicia faba) in Manitoba. Can. J. Plant Pathol., 7: 139-145.

[14] Maha, H. Mohamed; Gado, E. A. M.; El-Deeb, S. H. and Mostafa, M. H. (2014). Behavior of fungus Rhizoctonia solani under faba bean cotyledons when away from host and the effect of its starvation on aggressiveness. Journal of Yeast and Fungal Research 5(1): 1-8.

[15] Omar, S. A. M. (1986). Pathological studies on root rot diseases of faba bean (Vicia faba). Faba Bean Information Service (FABIS) Newsletter., 14: 34-37.

[16] Parmeter, J. R. Jr.; Snyder, W. C. and Reichle, R. E. (1963). Heterokaryosis and variability in plant pathogenic fungi. Annual Review of Phytopathology, 1: 51-76.

[17] Saitou,N., and Nei, M. (1987). The neighbor-joining method: a new method for reconstructing phylogenetic trees. Mol. Biol. Evol. 4 (4): 406-425.

[18] Salt, G. A. (1982). Factors affecting resistance to root rot and wilt diseaes. Pages 259-267 in G. C. Hawtin and C. Webb, eds., Faba Bean Improvement. Martinus Nijhoff Publishers, The Netherlands. 
[19] Sneh, B.; Burpee, L. and Ogoshi, A. (1991). Identification of Rhizoctonia Species. St Paul, MN, APS Press, pp. 133.

[20] Thompson, J.D.; Gibson, T.J.; Plewniak, F.; Jeanmougin, F. and Higgins, D.G. (1997). The ClustalX windows interface: flexible strategies for multiple sequence alignment aided by quality analysis tools. Nucleic Acids Research, 25: 4876-4882.

[21] Vilgalys, R. (1988). Genetic relatedness among anastomosis groups in Rhizoctonia as measured by DNA/DNA hybridization. Phytopathology 78:698-702.
[22] White, T. J.; Bruns, T.; Lee, S. and Taylor, J. W. (1990). Amplification and direct sequencing of fungal ribosomal RNA genes for phylogenetics. In: Innis, M.A., Gelfand, D.H., Sninsky, J.J. and White, T.J. (Eds.) PCR Protocols: A Guide to Methods and Applications. San Diego. Academic Press. pp. Pp. 315-322 